Additional file $\mathrm{E}$. The interview guide with patient and the public

\title{
Opening the discussion
}

- Greet the participants and thank them for taking part in the research.

- Explain again the purpose of my study.

- Ask participants if they would like to ask any question before starting the discussion.

- Emphasise participants that there is no right or wrong answer and that I am just interested in their experiences.

- Discuss the participant information sheet, if the participant has not read it in advance.

- Discuss the participant informed consent and ensure it is signed.

- Complete the patient's demographic form.

- Check the audio recorder and ask the participants if they are happy to begin the discussion.

Before we start this group discussion, I would like to confirm you know that:

- Your participation in this study is completely voluntary.

- You are free to withdraw but only up to the conclusion of the discussion.

- The discussion will be strictly confidential and anonymised and all information disclosed during this discussion will only be available to the research team. Excerpts from this discussion may be part of the final report of the project. However, information used in the project report will NOT be linked back to you. All reports and information collected will be stored securely at Newcastle University.

Are you ready to proceed with the discussion?

\section{Body of the discussion and research questions}

The questions will be about the transfer of care service, and the discussion will consist of four parts:

a. Patients' awareness of the electronic referral service.

b. Patients' acceptance/willingness to be referred to and interact with their community pharmacist post hospital discharge.

c. Patients' positive and negative experiences/views and expectations of using the eToC service and post-discharge community pharmacy services.

d. The difficulties and challenges associated with being unable to visit the $\mathrm{CP}$ and use the service.

a. Patients' awareness of the electronic referral service
1. What do you know about the transfer of care service where hospital inpatients are referred to their community pharmacy for care after they are discharged?

\section{Prompt:}

How much do you know about it? Can you tell me more?

2. How did you introduce/heard about it?

Prompt:

How do you think inpatients should be told about it? 


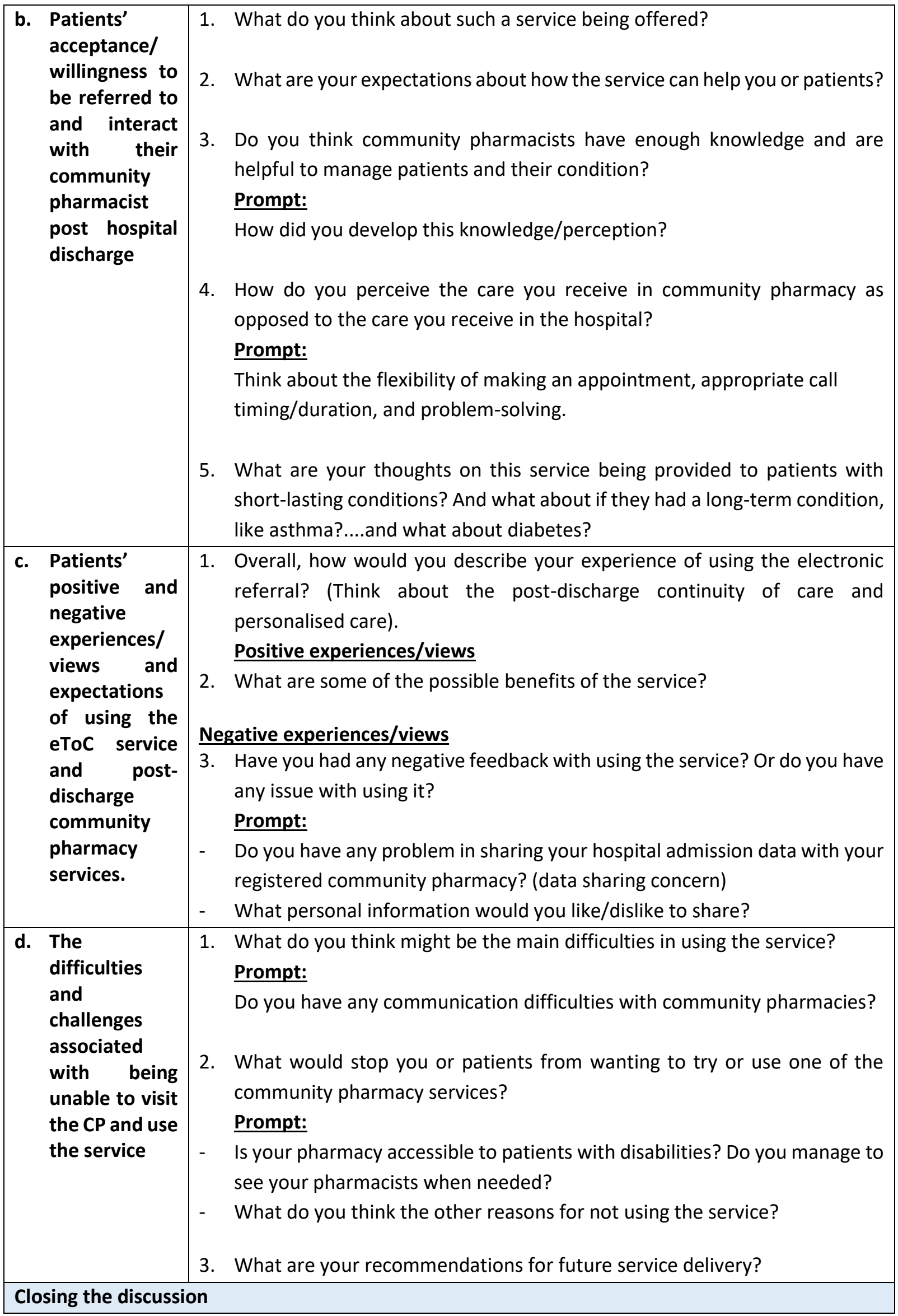


- $\quad$ Ask the participants if they would like to add or ask about anything else before closing the audio recorder and finishing the interview. Thank the participant again for taking part in the study. 